\title{
CARACTERIZACIÓN MORFOLÓGICA Y AGRONÓMICA DE ACCESOS EN YUCA EN LA REGION NOROESTE DEL RIO GRANDE DO SUL, BRASIL
}

\author{
J. KOEFENDER ${ }^{* 1}$, D. P. GOLLE ${ }^{1}$, C. E. MANFIO ${ }^{2}$, R. C. HORN ${ }^{1}$, A. SCHOFFEL ${ }^{3}$, J.A.R. de OLIVEIRA ${ }^{4}$, J.N. CAMERA ${ }^{1}$ \\ ${ }^{1}$ Universidade de Cruz Alta-RS, ${ }^{2}$ EPAGRI-SC, ${ }^{3}$ UFSM-RS, ${ }^{4}$ Eng. Agr. Autônomo \\ jkoefender@unicruz.edu.br ${ }^{*}$
}

Submetido 14/09/2018 - Aceito 07/11/2018

DOI: $10.15628 /$ holos.2018.7747

\section{RESUMEN}

El presente trabajo tiene como objetivo el evaluar las características morfológicas de 47 variedades tradicionales de yuca (Manihot esculenta), colectadas en propiedades rurales en la región del Alto Jacuí-RS. Fueron usadas estacas con cuatro yemas como semilla, en una área preparada de manera convencional, con arado de discos y rastrillo, dejando un espacio de 1,00 $\mathrm{x}$ $1,00 \mathrm{~m}$ entre surcos, siguiendo el línea experimental de bloques al azar, con tres repeticiones, constituidas estas por parcelas útiles de $5 \mathrm{~m}^{2}$ con 5 plantas cada una. Diez meses después de la siembra se evaluaron características morfológicas referentes a la parte aérea y radicular de la planta. La evaluación de los indicadores escogidos siguió la metodología de Fukuda \& Guevara (1998). Las variedades típicas de yuca de la región del Alto Jacuí, Rio Grande do Sul, conservados en el Banco de Germoplasma, presentan una elevada durabilidad genética, permitiendo su uso en el mejoramiento genético de la especie. Las características morfológicas de su parte aérea son diferentes si comparados con la mayoría de los descriptores morfológicos, a excepción de la pubescencia de la hoja joven y en el florecimiento. También predomina el color marrón oscuro en la película que cubre la raíz, color de la corteza radicular es blanca o crema, color de la pulpa de la raíz crema y la presencia de pedúnculo séssil en la mayoría de las variedades

PALABRAS-CLAVE: Manihot esculenta, características morfológicas, variabilidad genética.

\section{MORPHOLOGICAL AND AGRONOMIC CHARACTERIZATION OF CASSAVA ACCESSES IN NORTHWEST REGION OF RIO GRANDE DO SUL, BRASIL}

\begin{abstract}
The objective of this work was to evaluate the morphological characteristics of 47 cassava traditional accessions collected from rural properties in the Alto Jacuí-RS. The seedlings with four buds were planted in a conventionally prepared area with plowing and trenching with spacing of $1.00 \times 1.00 \mathrm{~m}$, following a randomized block design with three replications, consisting of useful plots of $5 \mathrm{~m}^{2}$, with 5 floors. Ten months after planting, the morphological characteristics of the aerial part and root of the plant were evaluated. The evaluation of the descriptors followed the methodology of Fukuda and Guevara (1998). The
\end{abstract}

accessions of cassava from the Alto Jacuí region, Rio Grande do Sul, conserved in the Germoplasm bank, show high genetic variability, which can be used in the genetic improvement of the crop. The morphological characteristics of the aerial part differ for most of the morphological descriptors, with the exception of young leaf pubescence and flowering. There is predominance of the color of the film of the dark brown root, color of the cortex of the white root or cream, color of the cream root pulp and the presence of sessile peduncle in the majority of evaluated accessions.

KEYWORDS: Manihot esculenta, morphological characters, genetic variability. 


\section{INTRODUCCIÓN}

La yuca (Manihot esculenta Crantz) es una especie domesticada de origen amazónica (Alves-Pereira, 2011). Sus raíces tuberosas, ricas en almidón (Figueiredo, 2012) son ampliamente utilizadas en la alimentación, en la producción de harina y almidón convencional para la industria de fécula, pudiendo también ser utilizada en la alimentación animal. Es una cultura de gran importancia para diversas poblaciones en el mundo (Figueiredo, 2012).

Según el Instituto Brasileño de Geografía y Estadística (IBGE), la producción brasileña de la raíz de yuca llegó a 23,71 millones de toneladas en el año de 2016, y a una área cultivada de 1,55 millones de hectáreas. En Brasil, variedades adaptadas a distintos locales son cultivadas en todas regiones del país (Brito et al., 2013), siendo que la diversificación de la especie es decurrente de la selección natural, lo que contribuye para la alta variabilidad genética existente (Barbosa et al., 2007). Las diferentes variedades son mantenidas en bancos de germoplasma, con la finalidad de mantener y preservar la diversidad genética de la especie y como base para programas de mejoramiento (Brito et al., 2013).

Sin embargo, debido a la escasez de datos botánicos sobre las inúmeras "variedades" brasileñas de yuca, se ha tornado necesario reunir distintos materiales para que sean evaluados en ensayos comparables, con el objetivo de obtener datos morfológicos capaces de generar indicaciones para un mejor manejo de esta especie (Albuquerque et al., 2009). Una manera de evaluar las características utilizadas con esta finalidad, es la evaluación a través del empleo de caracteres fenotípicos, como es el caso de los descriptores morfológicos. Según Vieira et al. (2009), esta caracterización es de fácil de medir y tiene bajo costo.

La variabilidad genética presente en las diferentes etnovariedades de esta especie indica características favorables para la conservación in situ y estudios de diversidad genética y evolución. Las plantas cultivadas, principalmente las denominadas etnovariedades, presentan una forma de recurso genético que debe ser preservado y conservado, pues podrá ser utilizado en programas de mejoramiento, especialmente en la transferencia de caracteres cualitativos (Faraldo et al., 2010). El trabajo tuvo como objetivo evaluar características morfológicas agronómicasde las raízes y de la parte aérea de 47 accessos tradicionales de yuca colectadas en la región del Alto Jacuí-RS.

\section{MATERIAL Y METODOS}

Se recogieron accesos tradicionales de yuca en ocho municipios de la región del Alto JacuíRS, en el año de 2013, totalizando 45 accesos y 2 variedades. Se consideran accesos tradicionales todo aquel material genético obtenido por medio de un sistema de cambio entre productores, o aquel mantenido por más de diez años en la propiedad por el agricultor, on farm.

Después de la obtención del material genético, se estableció, en el año agrícola de 2013/2014 y 2014/2015, un experimento en el Polo de Innovación Tecnológica del Alto Jacuí Campus de la Universidad de Cruz Alta, localizada geográficamente en la latitud $28^{\circ} 33^{\circ} 45,3^{\circ} \mathrm{S}$, 
longitud $53^{\circ} 37^{\circ} 20,3^{\circ} \mathrm{W}$ y altitud de $450 \mathrm{~m}$. Con un clima del tipo Cfa, de acuerdo con la clasificación de Köppen. El suelo está clasificado como Typic Hapludox (Soil Survey Staff, 2010), fue preparado de manera convencional, con un arado y un rastrillo, se utilizó un espaciamiento de 1,0 metro entre surcos y una profundidad de 0,10 metros.

La siembra se realizó en el mes de octubre, para los dos ciclos de cultivo. Las estacas usadas para semilla, recibieron cortes de aproximadamente $15 \mathrm{~cm}$ de largo (conteniendo 4 yemas vegetativas). La fertilicazión se realizó según las recomendaciones técnicas para esta especie, de acuerdo con los resultados del análisis de suelo (Arcilla 67\%, pH agua 5,7, Índice SMP 5,8, M.O. 4,0\%. P 13,8 mg/dm ${ }^{3}, \mathrm{~K} 200 \mathrm{mg} / \mathrm{dm}^{3}$, Aluminio $0,00 \mathrm{cmolc} / \mathrm{dm}^{3}, \mathrm{Ca} 4,0 \mathrm{cmolc} / \mathrm{dm} 3, \mathrm{Mg} 1,7$ cmolc $/ \mathrm{dm}^{3}, \mathrm{H}+\mathrm{Al} \mathrm{5,4} \mathrm{cmolc} / \mathrm{dm} 3, \mathrm{CTC}$ efectiva $6,2 \mathrm{cmolc} / \mathrm{dm}^{3}, \mathrm{CTC} \mathrm{pH} 7,011,6 \mathrm{cmolc} / \mathrm{dm}^{3}, \mathrm{~V} 53 \%$,

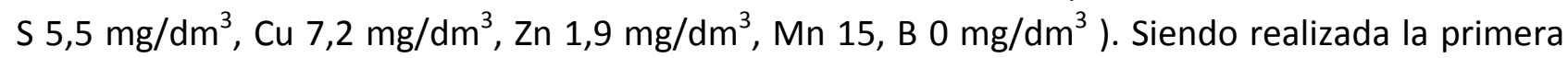
fertilización en el momento de la siembra y la segunda 30 días después. En el transcurrir del experimento fueron realizadas retiradas manuales como control de malezas.

El delineamiento experimental fue el de bloques al azar con tres repeticiones y 49 tratamientos, formados por 47 accesos de yuca junto con dos variedades registradas: Fepagro RS 13 (RS 13) y Fepagro RS 14 (RS14), siendo cada repetición compuesta por 5 plantas.

La evaluación de los indicadores escogidos siguió la metodología de Fukuda y Guevara (1998). Como indicadores observados en los tallos se utilizó: la coloración externa del tallo, de la corteza, de la epidermis y de los ramos, junto con su hábito de ramificación. Como indicadores para las hojas, se tuvieron en cuenta: la posición y la coloración del peciolo, la coloración del brote terminal, la presencia de pubescencia en la hoja joven, la forma del lóbulo, la distancia de entrenudos y la floración. En la raíz se tuvieron en cuenta: la coloración de la pulpa, la masa fresca de raíces (ton.ha ${ }^{-1}$ ), el diámetro de la raíz mayor $(\mathrm{cm})$, la longitud promedio de la raíz mayor $(\mathrm{cm})$, y la longitud promedio de la raíz menor $(\mathrm{cm})$, en dos cosechas.

Para caracterizar la parte aérea se utilizó como indicadores de tallo: la coloración externa del tallo, de la corteza, de la epidermis y de las ramas, junto con su manera de ramificarse. Como indicadores para las hojas, se tuvieron en cuenta: la posición y la coloración del peciolo, la coloración del brote terminal, la pubescencia de la hoja joven, la forma del lóbulo, la distancia de entrenudos y el florecimiento.

Los datos cuantitativos se sometieron al análisis de variancia y al análisis complementar comparados por el teste de Scott-Knott en $5 \%$ de probabilidad de error. Siendo usado el programa Sisvar 5.1 (Ferreira, 2008). Los demás fueron cuantificados y clasificados en porcentaje de acuerdo con sus respectivas categorías.

\section{RESULTADOS Y DISCUSIÓN}

\subsection{Descriptores de la raíz}

Cuando se evaluó el color de la película de la raíz, la mayor frecuencia fue el color marrón oscuro $(53,33 \%)$, seguido por el marrón claro $(24,44 \%)$ y por el blanco/crema $(17,78 \%)$, la menor frecuencia se dio para el color amarillo $(4,44 \%)$. En lo referente al color de la corteza de la raíz, el color blanco o crema tuvo una mayor predominancia, seguido del amarillo y del rosado. Con 
relación al color de la pulpa de la raíz, la mayor frecuencia se dio en el color crema (47\%), seguido por el blanco (35\%) y el amarillo (18\%). En lo referente a la presencia del péndulo, el mayor porcentaje ocurrió en el pedúnculo sésil (53\%), seguido por el de pedúnculo misto (38\%) y finalmente por la hoja pedunculada (9\%).

La variabilidad genética presente con relación a la coloración de la película, coloración de la corteza y coloración de la pulpa de la raíz, de los 47 accesos de yuca colectados, puede ser observada en la Planilla 1. Los accesos colectados en una misma ciudad pueden ser agrupados con características semejantes entre ellos, lo que indica una baja variabilidad genética.

El color externo y de la corteza de la raíz empezó a tener importancia en los últimos años, siendo que el ideal para la industria son los accesos que presenten una coloración blanca en su corteza y parte externa de la raíz. Chaib (2008) en estudios donde hacía evaluaciones de esta característica, observó que el $15,44 \%$ de los accesos presentaron color amarillo o rosado en la parte externa de la raíz y el $71,97 \%$ de ellos tenían el color del córtex blanca o crema; mientras que para la producción de yuca de mesa son preferibles las coloraciones externa de la raíz marrón claro u oscuro, siendo que el $83,85 \%$ de los accesos evidenciaron tales características. Datos semejantes a estos fueron encontrados en este trabajo, donde se observa la mayor predominancia de coloración externa de la raíz marrón claro u oscuro.

La predominancia del color de la película de la raíz marrón oscuro fue relatada por Barbosa (2013), donde hubo mayor predominancia de la coloración marrón oscura en un 66,67\% de los clones estudiados. Esa coloración no es la deseada por los agricultores de la región Sudoeste de Bahía (Fernandes et al., 2009). Los mismos autores afirman que tanto para la coloración externa de la raíz como para la corteza, la coloración clara tiene una mejor aceptación. Eso se debe a que al procesarse la yuca se hace un raspaje de la cáscara de forma manual y ocurre que algunos residuos permanecen, pudiendo así comprometer la calidad del producto final (Ramos, 2007). La predominancia de la coloración oscura en la región del Alto Jacuí, puede ser atribuida al hecho de que la mayoría de los productores cultiven la yuca para su propio consumo y no para la industria.

En relación a la coloración externa de la corteza, Barbosa (2013) también observó la predominancia de la coloración de la corteza blanca o crema, ambas características demuestran la preferencia por este color de raíz por el productor, cuando el objetivo es procesarlas. Vieira et al. (2008) afirman que los cultivos de yuca seleccionados para la industria presentan raíces con corteza blanca.

El color de la pulpa de la raíz es una de las características más importantes para el mejoramiento genético. Los agricultores tienen preferencia por variedades de mesa que presenten coloración de pulpa amarilla. En estudios realizados por Chaib (2009), se observó que un $18 \%$ de los accesos estudiados presentaban tal coloración; mientras que la mayoría presentó coloración de la pulpa de la raíz blanca, lo que es más interesante para la industria.

En trabajos realizados por Barbosa (2013), donde se estudiaba la presencia de pedúnculo, hubo una mayor predominancia del pedúnculo misto (51,89\%). Según Albuquerque et al. (2009) esa característica está relacionada con la facilidad de la cosecha. Entretanto, raíces pedunculadas 
Planilla 1: Caracterización morfológica de la parte radicular, referente a la coloración de la película, coloración de la corteza, coloración de la pulpa, presencia del pedúnculo en accesos de yuca de la región del Alto Jacuí, RS.

\begin{tabular}{|c|c|c|c|c|}
\hline \multicolumn{5}{|c|}{ Descriptores } \\
\hline Accesos & Película & Corteza & Pulpa & Pedúnculo \\
\hline CA02 & Marrón oscuro & Rosado & Crema & Mista \\
\hline CA03 & Marrón claro & Blanco/Crema & Blanco & Pedunculada \\
\hline CA04 & Marrón claro & Blanco/Crema & Crema & Mista \\
\hline CA05 & Marrón claro & Blanco/Crema & Crema & Sésil \\
\hline CA07 & Marrón oscuro & Blanco/Crema & Crema & Sésil \\
\hline CA08 & Marrón oscuro & Amarillo & Amarillo & Sésil \\
\hline CA09 & Marrón claro & Blanco/Crema & Blanco & Sésil \\
\hline FV01 & Marrón claro & Blanco/Crema & Blanco & Sésil \\
\hline FV03 & Amarillo & Amarillo & Blanco & Pedunculada \\
\hline FV05 & Marrón oscuro & Amarillo & Amarillo & Mista \\
\hline FV06 & Blanco/Crema & Blanco/Crema & Crema & Sésil \\
\hline FV07 & Marrón oscuro & Blanco/Crema & Crema & Sésil \\
\hline FV09 & Marrón oscuro & Blanco/Crema & Amarillo & Mista \\
\hline FV10 & Marrón claro & Blanco/Crema & Blanco & Sésil \\
\hline FV11 & Marrón oscuro & Blanco/Crema & Crema & Mista \\
\hline FV12 & Marrón oscuro & Blanco/Crema & Crema & Sésil \\
\hline FV13 & Blanco/Crema & Blanco/Crema & Crema & Sésil \\
\hline IB02 & Marrón claro & Amarillo & Crema & Mista \\
\hline IB03 & Marrón oscuro & Amarillo & Crema & Mista \\
\hline LCO2 & Marrón oscuro & Amarillo & Crema & Pedunculada \\
\hline LCO3 & Marrón oscuro & Amarillo & Amarillo & Mista \\
\hline LCO6 & Marrón oscuro & Blanco/Crema & Branco & Sésil \\
\hline SE06 & Marrón oscuro & Amarillo & Amarillo & Sésil \\
\hline SJ01 & Marrón oscuro & Blanco/Crema & Crema & Mista \\
\hline SJO2 & Marrón claro & Rosado & Blanco & Sésil \\
\hline SJ03 & Amarillo & Blanco/Crema & Crema & Sésil \\
\hline SJO4 & Marrón oscuro & Blanco/Crema & Blanco & Mista \\
\hline SJ06 & Marrón oscuro & Amarillo & Amarillo & Mista \\
\hline SJ07 & Marrón claro & Blanco/Crema & Blanco & Sésil \\
\hline SJ08 & Marrón claro & Blanco/Crema & Crema & Sésil \\
\hline SJ10 & Blanco/Crema & Blanco/Crema & Blanco & Mista \\
\hline TP01 & Blanco/Crema & Blanco/Crema & Blanco & Sésil \\
\hline TP02 & Blanco/Crema & Amarillo & Amarillo & Sésil \\
\hline TP03 & Blanco/Crema & Blanco/Crema & Crema & Sésil \\
\hline TP04 & Marrón escuro & Rosado & Blanco & Pedunculada \\
\hline TP05 & Marrón escuro & Blanco/Crema & Crema & Pedunculada \\
\hline TP06 & Marrón escuro & Blanco/Crema & Crema & Sésil \\
\hline XV01 & Blanco/Crema & Rosado & Blanco & Mista \\
\hline XV03 & Marrón oscuro & Blanco/Crema & Crema & Sésil \\
\hline XV04 & Marrón oscuro & Blanco/Crema & Amarillo & Sésil \\
\hline XV05 & Marrón oscuro & Blanco/Crema & Crema & Sésil \\
\hline XV08 & Blanco/Crema & Blanco/Crema & Blanco & Sésil \\
\hline XV09 & Marrón oscuro & Blanco/Crema & Crema & Mista \\
\hline XV10 & Marrón oscuro & Blanco/Crema & Blanco & Pedunculada \\
\hline XV11 & Marrón claro & Rosado & Blanco & Sésil \\
\hline RS 13 & Marrón claro & Blanco/Crema & Crema & Sésil \\
\hline RS 14 & Marrón oscuro & Blanco/Crema & Crema & Sésil \\
\hline
\end{tabular}

presentan mejor conservación pos-cosecha, pues el pedúnculo protege las raíces contra la podredumbre ocasionada por la menor exposición de la pulpa a los agentes patogénicos. 
Vieira et al. (2008), evaluando la variabilidad genética del banco de germoplasma de yuca da la Embrapa Cerrados, afirman que existe una preferencia de los pueblos que domesticaron la cultura de la yuca por algunas características como: raíces lisas, textura rugosa de la cáscara y color blanco de la pulpa. Sin embargo aunque se realicen cultivos de materiales con coloración de la pulpa más clara, se observa una preferencia, por parte de la población regional, por el consumo de harina amarilla. Esa coloración puede ser obtenida naturalmente a través de la utilización de yucas de raíces amarillas, o a través de un colorante artificial, siendo el Amarillo de Tartrazina el más frecuentemente utilizado. Según Gallego et al. (2007), la Tartrazina ha sido tradicionalmente utilizada para proveer el color amarillo a diversos productos, sin embargo tradicionalmente ha atraído la atención para posibles efectos alérgicos.

Ese escenario sugiere que algunos accesos puedan ser útiles como progenitores en programas de mejoramiento, una vez que gran número de estos presentan características deseables desde el punto de vista agronómico y fue detectada la presencia de elevada variabilidad genética entre ellos.

Cuando se evaluó la masa fresca $\left(\mathrm{kg}_{\text {. ha }}{ }^{-1}\right)$ en la cosecha 2013/2014, se observó una mayor variabilidad entre los accesos, hubo la formación de cuatro grupos, destacándose la variedad FV03 con un rendimiento de $45490 \mathrm{~kg} \cdot \mathrm{ha}^{-1}$. En la cosecha 2014/2015 hubo la formación de dos grupos entre los distintos accesos colectados en diferentes municipios de la región del Alto Jacuí-RS, destacándose la variedad RS13 con un rendimiento de 60000 kg.ha ${ }^{-1}$, y el acceso SJ02 (58000 $\left.\mathrm{kg} \cdot \mathrm{ha}^{-1}\right)$. Los accesos presentaron comportamiento diferente debido a condiciones climáticas distintas en las dos cosechas, lo que influenció directamente en el rendimiento (Planilla 2).

Para el diámetro de la mayor raíz, se observó en la cosecha 2013/2014 la formación de tres grupos, destacándose la variedad RS14 con un diámetro en la mayor raíz de 5,7 cm, siendo esta considerada como testigo, teniendo en seguida el acceso XV09 (5,66 cm). En la cosecha 2014/2015 se observó la formación de los dos grupos, destacándose el acceso CA06 con 6,0cm de ancho. El ancho promedio de raíz en la cosecha 2013/14 fue obtenido en el acceso TP 04 (59 cm) y en la cosecha 2014/15 en el acceso FV09 $(63,67 \mathrm{~cm})$, y el ancho de la menor raíz fue con el TP 02 en 2013/14 y CA 08 en 2014/15. Comparando todas las variables en ambas cosechas, se observó que los accesos CA02, CA05 SJ 03 y la variedad RS 14 presentaron los mayores valores, esta tendencia no fue observada solamente para la variable largura de la menor raíz (Planilla 2).

Según Enyi (1972), el número de vástagos flexibles tiene influencia en el rendimiento de raíces de la yuca. Las plantas que presentan un mayor número de vástagos, tienen un menor desarrollo de raíces si se les compara con plantas con sólo un vástago; esto debido a la competición entre el desarrollo de las raíces y de la parte aérea. La competición entre plantas de yuca por radiación solar, es debido al sombreado entre ellas mismas, lo que puede ocasionar el desarrollo exagerado de la parte aérea, la baja actividad fotosintética y consecuentemente en menores producciones (Enyi, 1973). Estudios relacionados al espaciamiento entre plantas podrían explicar esta variación entre a productividad de los distintos accesos evaluados en este estudio.

Variedades de yuca que presentan menor dosel pueden posibilitar mayores poblaciones de plantas, esto debido a una menor competición entre ellas por luz. Los lóbulos foliares estrechos 
Planilla 2: Masa fresca, diámetro de la mayor raíz (DMR cm), largura media de la mayor raíz (CMMAR cm) y largura media de la menor raíz (CMMER cm) añada 2013/14 y 2014/15 de accesos de yuca de la región del Alto Jacuí RS.

\begin{tabular}{|c|c|c|c|c|c|c|c|c|}
\hline \multirow[b]{2}{*}{ Accesos } & \multicolumn{2}{|c|}{ Masa fresca $\left(\mathrm{kg} \mathrm{ha}^{-1}\right)$} & \multicolumn{2}{|c|}{ DMR (cm) } & \multicolumn{2}{|c|}{ CMMAR (cm) } & \multicolumn{2}{|c|}{ CMMER (cm) } \\
\hline & $\begin{array}{l}\text { Cosecha } \\
2013 / 14\end{array}$ & $\begin{array}{l}\text { Cosecha } \\
2014 / 15\end{array}$ & $\begin{array}{l}\text { Cosecha } \\
2013 / 14\end{array}$ & $\begin{array}{l}\text { Cosecha } \\
2014 / 15\end{array}$ & $\begin{array}{l}\text { Cosecha } \\
2013 / 14\end{array}$ & $\begin{array}{l}\text { Cosecha } \\
2014 / 15 \\
\end{array}$ & $\begin{array}{l}\text { Cosecha } \\
2013 / 14\end{array}$ & $\begin{array}{l}\text { Cosecha } \\
2014 / 15 \\
\end{array}$ \\
\hline CA02 & $45270 a^{*}$ & $44000 \mathrm{a}$ & $5,34 \mathrm{a}$ & $5,00 \mathrm{a}$ & $45,0 \mathrm{a}$ & $47,4 \mathrm{a}$ & $12,6 \mathrm{c}$ & $10,2 \mathrm{c}$ \\
\hline CA03 & $5900 \mathrm{~d}$ & $22500 \mathrm{~b}$ & $2,98 \mathrm{~b}$ & $3,75 \mathrm{~b}$ & $36,9 \mathrm{~b}$ & $49,0 \mathrm{a}$ & $09,6 \mathrm{~d}$ & $17,7 \mathrm{c}$ \\
\hline CA04 & $9330 d$ & $28000 \mathrm{~b}$ & $2,02 \mathrm{c}$ & $3,60 \mathrm{~b}$ & $24,3 \mathrm{c}$ & 54,4 a & $11,5 \mathrm{~d}$ & $11,0 \mathrm{c}$ \\
\hline CA05 & 38090 a & 42000 a & 4,62 a & $6,00 \mathrm{a}$ & $51,4 a$ & $58,0 \mathrm{a}$ & $17,0 \mathrm{c}$ & $22,2 \mathrm{c}$ \\
\hline $\mathrm{CA} 07$ & $21950 \mathrm{c}$ & $24000 \mathrm{~b}$ & $4,47 a$ & $4,80 \mathrm{a}$ & $31,8 \mathrm{~b}$ & $40,0 \mathrm{~b}$ & $13,4 \mathrm{c}$ & $12,0 \mathrm{c}$ \\
\hline CA08 & $33900 \mathrm{~b}$ & $36000 \mathrm{a}$ & $5,22 a$ & $4,40 \mathrm{~b}$ & $44,6 \mathrm{a}$ & $47,6 \mathrm{a}$ & $17,1 \mathrm{c}$ & 42,6 a \\
\hline CA09 & $11110 \mathrm{~d}$ & $16000 \mathrm{~b}$ & $2,37 c$ & $4,00 \mathrm{~b}$ & $36,0 \mathrm{~b}$ & $48,6 \mathrm{a}$ & $09,9 \mathrm{~d}$ & $22,6 \mathrm{c}$ \\
\hline FV01 & 42170 a & $28000 \mathrm{~b}$ & $5,22 \mathrm{a}$ & $4,20 \mathrm{~b}$ & $42,9 a$ & $55,0 \mathrm{a}$ & $12,1 \mathrm{~d}$ & $16,6 \mathrm{c}$ \\
\hline FV03 & 45490 a & $42000 \mathrm{a}$ & $4,74 \mathrm{a}$ & $5,00 \mathrm{a}$ & $52,0 \mathrm{a}$ & $56,0 \mathrm{a}$ & $16,2 \mathrm{c}$ & $30,2 \mathrm{~b}$ \\
\hline FV05 & $8100 \mathrm{~d}$ & $24000 \mathrm{~b}$ & $1,92 \mathrm{c}$ & $4,20 \mathrm{~b}$ & $22,6 \mathrm{c}$ & $39,4 \mathrm{~b}$ & 06,4 e & $12,2 \mathrm{c}$ \\
\hline FV06 & 36635 a & $20000 \mathrm{~b}$ & $5,15 \mathrm{a}$ & $3,80 \mathrm{~b}$ & $40,1 \mathrm{a}$ & $33,4 \mathrm{~b}$ & $10,4 \mathrm{~d}$ & $11,6 \mathrm{c}$ \\
\hline FV07 & $14150 \mathrm{~d}$ & $34000 \mathrm{~b}$ & $4,24 \mathrm{a}$ & $4,60 \mathrm{a}$ & $39,0 \mathrm{a}$ & $52,8 \mathrm{a}$ & $11,4 \mathrm{~d}$ & $20,4 \mathrm{c}$ \\
\hline FV09 & $30785 \mathrm{~b}$ & $46666 \mathrm{a}$ & $4,22 a$ & $5,10 a$ & $42,2 \mathrm{a}$ & $63,6 \mathrm{a}$ & $14,1 \mathrm{c}$ & $12,3 \mathrm{c}$ \\
\hline FV10 & $26520 \mathrm{~b}$ & $34000 \mathrm{~b}$ & $4,17 \mathrm{a}$ & $4,00 \mathrm{~b}$ & $47,2 \mathrm{a}$ & $44,8 \mathrm{~b}$ & $15,0 \mathrm{c}$ & $17,8 \mathrm{c}$ \\
\hline FV11 & $18460 \mathrm{c}$ & $22000 \mathrm{~b}$ & $3,63 \mathrm{~b}$ & $3,60 \mathrm{~b}$ & $28,1 \mathrm{~b}$ & $49,0 \mathrm{a}$ & $08,9 d$ & $14,6 \mathrm{c}$ \\
\hline FV12 & $8850 \mathrm{~d}$ & $43333 \mathrm{a}$ & $1,47 \mathrm{c}$ & 4,67 a & $8,70 \mathrm{~d}$ & $48,6 \mathrm{a}$ & $05,1 \mathrm{e}$ & $17,3 \mathrm{c}$ \\
\hline FV13 & $18010 \mathrm{c}$ & $50000 \mathrm{a}$ & $4,32 \mathrm{a}$ & $5,40 \mathrm{a}$ & $40,5 \mathrm{a}$ & $58,0 \mathrm{a}$ & $19,1 \mathrm{~b}$ & $09,8 \mathrm{c}$ \\
\hline IB02 & $25880 \mathrm{~b}$ & $27500 \mathrm{~b}$ & $4,07 \mathrm{a}$ & $4,00 \mathrm{~b}$ & 47,4 a & $45,0 \mathrm{~b}$ & $16,0 \mathrm{c}$ & $16,2 \mathrm{c}$ \\
\hline IB03 & $31730 \mathrm{~b}$ & $26000 \mathrm{~b}$ & $4,76 \mathrm{a}$ & $3,80 \mathrm{~b}$ & $48,9 \mathrm{a}$ & $40,0 \mathrm{~b}$ & $11,6 \mathrm{~d}$ & $14,4 \mathrm{c}$ \\
\hline LC01 & $17050 \mathrm{c}$ & - & $3,12 \mathrm{~b}$ & - & $25,1 \mathrm{c}$ & - & 07,8 e & - \\
\hline LCO2 & $24900 \mathrm{~b}$ & $46000 \mathrm{a}$ & $2,92 \mathrm{~b}$ & $4,60 \mathrm{a}$ & $20,9 \mathrm{c}$ & $54,4 \mathrm{a}$ & 05,7 e & $14,2 \mathrm{c}$ \\
\hline LCO3 & $11660 \mathrm{~d}$ & $30000 \mathrm{~b}$ & $2,20 \mathrm{c}$ & $5,00 \mathrm{a}$ & $22,7 \mathrm{c}$ & $27,3 \mathrm{~b}$ & $12,6 \mathrm{c}$ & $20,0 \mathrm{c}$ \\
\hline LC06 & 19785 c & $20000 \mathrm{~b}$ & $4,50 \mathrm{a}$ & $4,00 \mathrm{~b}$ & $50,4 \mathrm{a}$ & $34,0 \mathrm{~b}$ & $22,1 \mathrm{a}$ & $19,0 \mathrm{c}$ \\
\hline SE06 & $32120 \mathrm{~b}$ & $25000 \mathrm{~b}$ & $5,33 \mathrm{a}$ & $5,00 \mathrm{a}$ & $44,1 \mathrm{a}$ & $41,5 \mathrm{~b}$ & $16,0 \mathrm{c}$ & $12,5 \mathrm{c}$ \\
\hline SJ01 & $12485 d$ & $22000 \mathrm{~b}$ & $3,43 \mathrm{~b}$ & $5,00 \mathrm{a}$ & $31,6 \mathrm{~b}$ & $40,6 \mathrm{~b}$ & $10,5 \mathrm{~d}$ & $11,6 \mathrm{c}$ \\
\hline SJ02 & $33090 \mathrm{~b}$ & 58000 a & $5,47 \mathrm{a}$ & $5,20 \mathrm{a}$ & $52,6 \mathrm{a}$ & $57,2 \mathrm{a}$ & $12,8 \mathrm{c}$ & $12,6 \mathrm{c}$ \\
\hline $\mathrm{SJO3}$ & $43430 \mathrm{a}$ & $44000 \mathrm{a}$ & $4,49 a$ & $5,20 \mathrm{a}$ & $55,8 \mathrm{a}$ & $58,0 \mathrm{a}$ & $13,3 \mathrm{c}$ & $16,6 \mathrm{c}$ \\
\hline SJ04 & $26808 \mathrm{~b}$ & $24000 \mathrm{~b}$ & $4,53 \mathrm{a}$ & $4,20 \mathrm{~b}$ & $55,2 \mathrm{a}$ & $47,8 \mathrm{a}$ & $14,9 \mathrm{c}$ & $15,6 \mathrm{c}$ \\
\hline SJ06 & $24510 \mathrm{~b}$ & $40000 \mathrm{a}$ & $4,64 \mathrm{a}$ & $3,60 \mathrm{~b}$ & $33,8 \mathrm{~b}$ & $46,8 \mathrm{a}$ & $10,6 d$ & $12,0 \mathrm{c}$ \\
\hline SJ07 & $29575 b$ & $30000 \mathrm{~b}$ & 4,79 a & $4,20 \mathrm{~b}$ & $35,4 \mathrm{~b}$ & $42,4 \mathrm{~b}$ & $15,2 \mathrm{c}$ & $16,2 \mathrm{c}$ \\
\hline SJ08 & $23370 \mathrm{c}$ & $32000 \mathrm{~b}$ & $4,30 a$ & $5,20 \mathrm{a}$ & $35,0 \mathrm{~b}$ & 57,8 a & $13,8 \mathrm{c}$ & $19,2 \mathrm{c}$ \\
\hline SJ10 & $31600 \mathrm{~b}$ & 38000 a & $4,88 a$ & $4,00 \mathrm{~b}$ & $45,9 a$ & $58,0 \mathrm{a}$ & $12,2 \mathrm{~d}$ & $15,2 \mathrm{c}$ \\
\hline TP01 & 38620 a & $18000 \mathrm{~b}$ & $4,91 \mathrm{a}$ & $4,00 \mathrm{~b}$ & $45,6 \mathrm{a}$ & $33,0 \mathrm{~b}$ & $17,4 \mathrm{c}$ & $12,4 \mathrm{c}$ \\
\hline TP02 & $12135 d$ & $16000 \mathrm{~b}$ & $3,62 \mathrm{~b}$ & $4,40 \mathrm{~b}$ & $42,4 \mathrm{a}$ & $37,8 \mathrm{~b}$ & $25,8 \mathrm{a}$ & $15,6 \mathrm{c}$ \\
\hline TP03 & 20790 c & 38000 a & $3,93 \mathrm{a}$ & $5,40 \mathrm{a}$ & $35,1 \mathrm{~b}$ & $43,2 \mathrm{~b}$ & $14,8 \mathrm{c}$ & $18,4 \mathrm{c}$ \\
\hline TP04 & $46050 \mathrm{a}$ & $22000 \mathrm{~b}$ & $5,38 \mathrm{a}$ & $3,60 \mathrm{~b}$ & $57,0 \mathrm{a}$ & $50,0 \mathrm{a}$ & $17,3 \mathrm{c}$ & $28,6 \mathrm{~b}$ \\
\hline TP05 & 20830 c & $28000 \mathrm{~b}$ & $5,42 a$ & $4,60 \mathrm{a}$ & $41,9 a$ & $49,2 \mathrm{a}$ & $19,0 \mathrm{~b}$ & $14,8 \mathrm{c}$ \\
\hline TP06 & $19050 \mathrm{c}$ & $28000 \mathrm{~b}$ & $4,97 \mathrm{a}$ & $4,60 \mathrm{a}$ & $38,8 \mathrm{a}$ & $34,00 \mathrm{~b}$ & $14,0 \mathrm{c}$ & $09,8 \mathrm{c}$ \\
\hline XV01 & 36830 a & $26000 \mathrm{~b}$ & $4,93 a$ & $3,60 \mathrm{~b}$ & 49,9 a & 53,00 a & $13,3 \mathrm{c}$ & $14,6 \mathrm{c}$ \\
\hline XV02 & $25420 \mathrm{~b}$ & - & $4,95 a$ & - & $37,4 \mathrm{~b}$ & - & $15,1 \mathrm{c}$ & - \\
\hline XV03 & $25645 b$ & $16000 \mathrm{~b}$ & $4,00 \mathrm{a}$ & $4,00 \mathrm{~b}$ & $29,0 \mathrm{~b}$ & $37,6 \mathrm{~b}$ & $09,3 \mathrm{~d}$ & $13,4 \mathrm{c}$ \\
\hline XV04 & $27370 \mathrm{~b}$ & $30000 \mathrm{~b}$ & 3,99 a & $4,50 \mathrm{a}$ & 54,7 a & $49,0 \mathrm{a}$ & $15,2 \mathrm{c}$ & $15,0 \mathrm{c}$ \\
\hline XV05 & $33520 \mathrm{~b}$ & $28000 \mathrm{~b}$ & $5,64 \mathrm{a}$ & $3,80 \mathrm{~b}$ & $45,9 a$ & $48,4 \mathrm{a}$ & $16,3 \mathrm{c}$ & $13,3 \mathrm{c}$ \\
\hline XV08 & $21270 \mathrm{c}$ & $20000 \mathrm{~b}$ & $4,45 a$ & $3,60 \mathrm{~b}$ & $37,9 a$ & $40,6 \mathrm{~b}$ & $18,3 \mathrm{~b}$ & $17,0 \mathrm{c}$ \\
\hline XV09 & $28080 \mathrm{~b}$ & $32000 \mathrm{~b}$ & $5,66 \mathrm{a}$ & $3,60 \mathrm{~b}$ & 39,6 a & $49,2 \mathrm{a}$ & $13,4 \mathrm{c}$ & $11,2 \mathrm{c}$ \\
\hline XV10 & $05760 \mathrm{~d}$ & $25000 \mathrm{~b}$ & $2,04 \mathrm{c}$ & $4,50 \mathrm{a}$ & $26,9 \mathrm{~b}$ & $50,5 \mathrm{a}$ & 04,6 e & $22,0 \mathrm{c}$ \\
\hline XV11 & $22830 \mathrm{c}$ & $34000 \mathrm{~b}$ & $3,29 \mathrm{~b}$ & $3,80 \mathrm{~b}$ & $35,0 b$ & $45,4 \mathrm{~b}$ & $10,7 d$ & $15,8 \mathrm{c}$ \\
\hline RS 13 & $32040 \mathrm{~b}$ & $60000 \mathrm{a}$ & $4,89 a$ & $5,30 \mathrm{a}$ & $51,1 \mathrm{a}$ & $52,4 \mathrm{a}$ & $15,2 \mathrm{c}$ & $11,0 \mathrm{c}$ \\
\hline RS 14 & $40340 \mathrm{a}$ & $36000 \mathrm{a}$ & $5,70 \mathrm{a}$ & $5,30 \mathrm{a}$ & $47,3 \mathrm{a}$ & $50,6 \mathrm{a}$ & $13,2 \mathrm{c}$ & $16,0 \mathrm{c}$ \\
\hline CV \% & 52 & 30 & 15 & 37 & 39 & 23 & 48 & 45 \\
\hline
\end{tabular}

* Los promedios seguidos de la misma letra en la columna, no difieren entre ellos por el test de Scott-knott, en $5 \%$ de probabilidad. 
permiten un menor efecto de sombra entre las hojas de la misma planta, permitiendo una mejor distribución y utilización de la radiación solar (Williams \& Ghazali, 1969).

Aunque en este trabajo no se tuvo en cuenta el indicador número de raíces, Takahashi \& Guerini (1998) relatan que el rendimiento parece no estar relacionado con este indicador, sino con su tamaño. El peso de las raíces se relaciona de manera linear con su diámetro y longitud.

Debido a la gran variación en la longitud de las raíces comerciales de la yuca de mesa, la Compañía de Entrepuestos y Almacenes Generales de São Paulo tiene a esta característica como el factor más importante en su clasificación. En un trabajo realizado por Williams (1974), se encontraron relaciones lineares entre la cantidad promedio de masa y el diámetro de las raíces, así mayor el diámetro de las raíces, mayor es el rendimiento promedio de las raíces por planta. Esto también pudo ser comprobado en este trabajo, pues la mayoría de los accesos evaluados presentaron una relación entre rendimiento, diámetro y longitud de la raíz.

Para la producción de raíces de yuca de mesa se tiene a la cantidad de masa promedio de las raíces, como un indicador importante, pues esta se relaciona directamente con el tamaño de las raíces, así al entrar en la clasificación de grandes hacen posible el aumento de los proventos económicos para los productores rurales. Ahora, cuando se trata de raíces para fines industriales, el índice de masa promedio de las raíces pierde relevancia, ya que la remuneración del agricultor se dará en función del total producido. Aún así, raíces mayores son preferidas por las industrias de una manera general, pues promueven un mayor rendimiento industrial, con menores pérdidas en el momento de retirar la cáscara. Raíces mayores pueden aún contener mayores cantidades de materia seca, lo que posee una elevada relación con los contenidos de almidón (Aguiar, 2003).

Otro factor importante que puede influenciar en las variables evaluadas en este trabajo es la hipótesis confirmada por Alves (2006), donde se expone que en el momento en que las plantas están bajo la sombra, ocurre una limitación de la fotosíntesis y gran parte de los foto asimilados es destinada para el crecimiento de la parte aérea, disminuyendo el crecimiento de las raíces de reserva, indicando que los tallos y las hojas drenan energía de la planta con más fuerza que las propias raíces. Así, una estrategia de manejo para que se obtenga una mayor productividad de raíces, con estas variedades en cultivos ya listos en Rio Grande do Sul, sería optar por un espaciamiento mayor que el de 0,8 $\times$ 0,8m. Por otro lado, al existir un interés por parte del agricultor en usar la parte aérea para el tratamiento animal, la siembra temprana de esta variedad permite el aumento en el crecimiento de la parte aérea, sin reducción significativa de la productividad de sus raíces. Una vez más, se destaca el espaciamiento y el ciclo de cada variedad como factores que deberán ser analizados en futuros estudios.

Los datos obtenidos en este trabajo están de acuerdo con los obtenidos por Tironi et al. (2015) donde los cultivares Fepagro-RS13 y Fepagro-RS14, presentaron elevada producción de raíces tuberosas (31000 kg.ha ${ }^{-1}$ ), aunque en este estudio la producción fue superior. Esto es un indicativo de que esas dos variedades pueden ser consideradas de doble propósito (producción de parte aérea y de raíces), con una buena adaptación a las condiciones de cultivo en el ambiente subtropical característico en esta región del Rio Grande do Sul (Chielle et al., 2007, 2009) y, así, tenerse como una buena opción para el agricultor familiar gaucho. 


\subsection{Descriptores de la parte aérea}

Dentro de los 47 accesos de yuca usados en este trabajo, se pudo verificar una gran variabilidad genética, tales como el color externo del tallo, de la corteza, de la epidermis y de las ramas, así como en lo referente al hábito de ramificación, de posición y de color del peciolo, color del brote terminal, de la pubescencia de la hoja joven, de la forma del lóbulo y de la distancia entrenudos (Planilla 3). Se pudo verificar el florecimiento en todos los accesos y las variedades estudiadas, así como la ausencia de la pubescencia en las hojas jóvenes.

Con referencia al indicador color externo del tallo, los accesos se agruparon en cinco clases, con base en la metodología de Fukuda \& Guevara (1998). La mayor frecuencia en el color externo del tallo fue para la color gris (31,91\%), seguido por el plateado (19,15\%), el marrón claro $(19,15 \%)$, el verde amarillento $(12,77 \%)$, el dorado $(10,64 \%)$ y se tuvo la menor frecuencia con el color marrón oscuro (6,38\%). Según Barbosa (2013), el color externo del tallo de los accesos de yuca presentan una gran variabilidad, misma que puede ser explicada debido a que esta característica no tiene ningún interés económico, siendo así no son tenidos en cuenta dentro de los procesos de selección. Sin embargo, son interesantes para el agricultor pues sirven como un parámetro para diferenciar raíces de diferentes cultivares. Para el indicador color de la corteza, los datos observados en los accesos tradicionales estudiados, fueron de 57,45\% para el color verde claro y $42,55 \%$ para el verde oscuro (Planilla 3 ).

Con referencia al color de la epidermis, se observó que el color marrón claro presentó mayor la frecuencia (51,06\%), la 2 (25,53\%), la 1 (21,18\%) y el color naranja (2,13\%). En estudios realizados por Nolasco (2011) con materiales de la región nordeste del Brasil, la coloración 2 también fue observada en los cultivares 2009.02, 2009.25, 2009.30, 2009.48, 2009.57 y 2009. De acuerdo con Ramos (2007), la coloración de la epidermis del tallo, es una característica que puede variar entre los diferentes genotipos y con la edad de la planta, siendo así, en la fase juvenil pueden ocurrir diferentes tonalidades tales como el rojo claro, el pardo y el verde. Es importante decir que los aspectos edafoclimáticos y prácticas de cultivo también pueden interferir en el color de la epidermis del tallo, pues afectan la disponibilidad de los nutrientes del suelo y cambian la asimilación de estos por las plantas.

Se verificó un mayor porcentaje de accesos tradicionales con el color verde de las ramas (51,06\%), seguido por el color verde morado $(40,40 \%)$ y el morado $(8,51 \%)$.

Para la característica hábito de ramificación la mayor frecuencia fue observada para la ramificación tricotómico $(72,34 \%)$, seguido por el dicotómico $(17,02 \%)$ y el erecto $(10,64 \%)$. En lo relativo a la posición del pecíolo, las datos indicaron: inclinado para arriba $(63,83 \%)$, de forma irregular $(19,15 \%)$ y en posición horizontal $(17,02 \%)$. Los hábitos de ramificación dicotómico y tricotómico de las plantas es una característica deseable, pues cuanto mayor el número de ramos, mayor será la contribución para la producción de las estacas semilla, mismas que serán importantes para el establecimiento de un nuevo ciclo de cultivo. (Barbosa, 2013). Sin embargo la característica de ramificación recta hace más fácil la cosecha de las ramas, además de formar un cultivo con copa uniforme, además de hacer más fácil las labores de cultivo. Según Ramos (2007) 
el hábito de ramificación es una característica de extrema importancia para la comercialización de raíces de yuca.

Con relación al color del peciolo, se obtuvo una predominancia dentro del color verde $(35,44 \%)$. Ahora, para el color del rebrote terminal se observó la formación de tres grupos, siendo que la mayoría de los accesos $(76,60 \%)$ está dentro del grupo considerado como dentro de un color verde con tonalidad morada.

La predominancia de materiales vegetales de yuca con coloración dentro de un color verde con tonalidad morada, puede tener relación con la presencia del pigmento llamado antocianina. Las antocianinas son substancias pertenecientes al grupo de flavonoides y son responsables por colores que pueden variar del salmón rosado al rojo y del fucsia para el azul oscuro, en frutos, flores y hojas de las angiospermas (Andersen \& Jordheim, 2006). Conforme Suresh et al. (2011), después de la clorofila, las antocianinas pertenecen al grupo más importante de pigmentos visibles de las plantas. Este pigmento tiene una propiedad antioxidante y puede actuar también como un soluto compatible para el ajuste osmótico, en época de sequía (Andersen \& Jordhein, 2006). Según Chalker-Scott (1999) los tejidos vegetales que contienen antocianinas son más resistentes al déficit hídrico, siendo la coloración morada considerada como una característica evolutiva de adaptación al estrés hídrico, tanto en bióticos como en abióticos.

La presencia de pubescencia en las plantas jóvenes, fue una característica que se observó en el $100 \%$ de las plantas evaluadas, así como la floración y la presencia de frutos. En el indicador forma del lóbulo, los datos obtenidos en los accesos se distribuyeron así, de forma lanceolada $(34,04 \%)$, de forma oblongo-lanceolada $(21,28 \%)$, de forma recta o linear $(21,28 \%)$, de forma linear-pandurada (12,77\%), elíptico-lanceolada (10,64\%). En este estudio, así como en los datos encontrados por Barbosa (2013), la forma lanceolada del lóbulo ocurrió en la mayoría de los accesos estudiados.

Se pudo verificar que el $55,32 \%$ de los accesos tradicionales presentaron una distancia de entrenudos promedio de $8-15 \mathrm{~cm}$ y $44,68 \%$ con una anchura $\geq 15 \mathrm{~cm}$; aparte de presentar un $100 \%$ de florecimiento.

La caracterización de los accesos de yuca por medio de datos morfológicos busca básicamente estudiar la diferenciación fenotípica (Almeida et al., 1993; Araujo et al., 2005). Esta gran variabilidad verificada entre los diferentes cultivares estudiados, puede ser explicada por el hecho de que los pequeños agricultores siempre han mantenido una elevada variabilidad genética. Esto debido al cultivo de un gran número de genotipos de manera asociada y con la constante introducción de nuevos genotipos (Sambatti et al., 2000). Otro hecho que puede estar asociado a la grande variabilidad genética, se debe a que los productores familiares de yuca mantienen un gran número de variedades en sus propiedades, incluso las menos productivas (Fregene et al., 2003).

Conforme Elias et al. (2001) el cultivo de yuca puede generar recombinantes que se propagan a través de semillas que, cuando incorporados al sistema de cultivo y mantenido por propagación asexual, pueden incrementar la base genética. 
Planilla 3: Caracterización morfológica de la parte aérea, referente a la coloración externa del tallo (CEC), coloración de la corteza (CÓR), coloración de la epidermis (EPI), coloración de los ramos (RAM), peciolo, coloración del peciolo (PEC), coloración del rebrote terminal (BRT), habito de ramificación, posición del peciolo, forma del lóbulo y distancia de entrenudos de $\mathbf{4 7}$ accesos de yuca tradicional de la región del Alto Jacuí, RS.

\begin{tabular}{|c|c|c|c|c|c|c|c|c|c|c|}
\hline \multirow[b]{2}{*}{ Accesos } & \multicolumn{10}{|c|}{ Descriptores } \\
\hline & CEC & CÓR & EPI & RAM & PEC & BRT & $\begin{array}{l}\text { Hábito de } \\
\text { ramificación }\end{array}$ & $\begin{array}{c}\text { Posición } \\
\text { del } \\
\text { pecíolo }\end{array}$ & $\begin{array}{c}\text { Forma } \\
\text { del } \\
\text { lóbulo }\end{array}$ & $\begin{array}{l}\text { Distancia } \\
\text { entrenudos }\end{array}$ \\
\hline \multicolumn{11}{|c|}{ 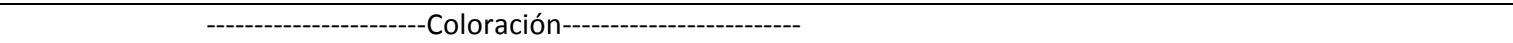 } \\
\hline CA02 & $\mathrm{C}^{*}$ & Ve & $\mathrm{Mc}$ & $\mathrm{Va}$ & $\mathrm{V}$ & Vc & $\mathrm{D}$ & 1 & $\mathrm{El}$ & $\mathrm{M}$ \\
\hline CA03 & $\mathrm{Mc}$ & Ve & $\mathrm{Mc}$ & Va & Vav & Va & D & IC & $\mathrm{Ol}$ & $M$ \\
\hline CA04 & $\mathrm{P}$ & Vc & C & V & Vav & $\mathrm{Va}$ & $\mathrm{T}$ & 1 & L & $\mathrm{L}$ \\
\hline CA05 & Mc & Vc & $\mathrm{Mc}$ & V & Vav & Vc & $\mathrm{T}$ & 1 & L & $\mathrm{L}$ \\
\hline CA07 & C & Vc & $\mathrm{Me}$ & V & Vam & $\mathrm{Va}$ & $\mathrm{E}$ & IC & R ou $L$ & $M$ \\
\hline CA08 & C & Ve & $\mathrm{Mc}$ & $\mathrm{R}$ & Ves & $\mathrm{Va}$ & $\mathrm{T}$ & $\mathrm{H}$ & R ou $\mathrm{L}$ & $M$ \\
\hline CA09 & $\mathrm{Mc}$ & Vc & $\mathrm{Mc}$ & $\mathrm{Va}$ & Vav & $\mathrm{Va}$ & D & IC & $\mathrm{Lp}$ & $M$ \\
\hline FV01 & D & Ve & $\mathrm{Mc}$ & V & Vam & $\mathrm{R}$ & $T$ & 1 & $\mathrm{~L}$ & $M$ \\
\hline FV03 & $\mathrm{Va}$ & Vc & C & V & Vav & $\mathrm{Va}$ & $T$ & 1 & $\mathrm{~L}$ & $\mathrm{~L}$ \\
\hline FV05 & C & Vc & $\mathrm{Mc}$ & $\mathrm{Va}$ & Vav & $\mathrm{Va}$ & $\mathrm{T}$ & IC & Ol & $\mathrm{L}$ \\
\hline FV06 & Va & Vc & C & V & Vav & Vc & D & 1 & R ou L & $M$ \\
\hline FV07 & C & Ve & $\mathrm{Me}$ & Va & V & $\mathrm{Va}$ & $\mathrm{T}$ & IC & Lp & $M$ \\
\hline FV09 & $\mathrm{Me}$ & Ve & $\mathrm{Me}$ & $\mathrm{Va}$ & Ves & $\mathrm{Va}$ & $T$ & IC & Ol & $\mathrm{L}$ \\
\hline FV10 & $P$ & Vc & C & V & Vav & $\mathrm{Va}$ & $\mathrm{T}$ & IC & $\mathrm{L}$ & $\mathrm{L}$ \\
\hline FV11 & D & Vc & $\mathrm{Mc}$ & V & Vav & $\mathrm{Va}$ & $\mathrm{T}$ & I & R ou $\mathrm{L}$ & $M$ \\
\hline FV12 & C & Ve & $\mathrm{Me}$ & Va & V & $\mathrm{Va}$ & $\mathrm{T}$ & IC & $\mathrm{Ol}$ & $\mathrm{L}$ \\
\hline FV13 & $\mathrm{Va}$ & Vc & C & V & Vam & $\mathrm{Va}$ & $\mathrm{T}$ & Ic & Ol & $\mathrm{L}$ \\
\hline IB02 & $P$ & Ve & $\mathrm{Me}$ & V & Vam & $\mathrm{Va}$ & $\mathrm{T}$ & IC & L & $\mathrm{L}$ \\
\hline IB03 & $\mathrm{Mc}$ & Vc & $\mathrm{Me}$ & V & Vam & $\mathrm{Va}$ & $\mathrm{T}$ & IC & $\mathrm{Ol}$ & $M$ \\
\hline LC01 & $P$ & Vc & $\mathrm{Mc}$ & $\mathrm{R}$ & V & $\mathrm{Va}$ & $\mathrm{E}$ & $\mathrm{H}$ & L & $M$ \\
\hline LCO2 & $\mathrm{Me}$ & Ve & $\mathrm{Mc}$ & $\mathrm{Va}$ & v & $\mathrm{Va}$ & $T$ & IC & Ol & $\mathrm{L}$ \\
\hline LCO3 & C & Ve & $\mathrm{Mc}$ & V & Ves & $\mathrm{Va}$ & $T$ & $\mathrm{H}$ & Ol & $\mathrm{L}$ \\
\hline LC06 & D & Vc & $\mathrm{Mc}$ & V & Vav & Vc & $\mathrm{T}$ & IC & L & $M$ \\
\hline SE06 & C & Ve & $\mathrm{Mc}$ & $\mathrm{Va}$ & V & $\mathrm{Va}$ & $T$ & IC & Ol & $\mathrm{L}$ \\
\hline SJ01 & $\mathrm{Mc}$ & Ve & $\mathrm{Mc}$ & V & Vam & $\mathrm{Va}$ & $\mathrm{T}$ & IC & R ou $L$ & $M$ \\
\hline SJ02 & $C$ & $\mathrm{Ve}$ & $\mathrm{Me}$ & $\mathrm{Va}$ & V & Vc & $\mathrm{T}$ & IC & $\mathrm{El}$ & $\mathrm{L}$ \\
\hline $\mathrm{SJ} 03$ & $\mathrm{Va}$ & Vc & C & V & Vav & $\mathrm{Va}$ & $T$ & 1 & L & $M$ \\
\hline SJ04 & $\mathrm{Mc}$ & Vc & $\mathrm{Mc}$ & $\mathrm{Va}$ & Ves & $\mathrm{Va}$ & $\mathrm{T}$ & I & Ol & $\mathrm{L}$ \\
\hline SJ06 & $P$ & Ve & La & Va & Ves & $\mathrm{Va}$ & $\mathrm{T}$ & IC & Ol & $\mathrm{L}$ \\
\hline SJ07 & $\mathrm{Mc}$ & Vc & $\mathrm{Mc}$ & Va & Vav & $\mathrm{Va}$ & $\mathrm{T}$ & IC & Lp & $M$ \\
\hline SJ08 & C & Ve & $\mathrm{Mc}$ & V & Vav & $\mathrm{Va}$ & $T$ & $\mathrm{H}$ & Lp & $M$ \\
\hline SJ10 & $\mathrm{Va}$ & Vc & C & V & Vav & $\mathrm{Va}$ & $\mathrm{T}$ & IC & L & $M$ \\
\hline TP01 & D & Vc & C & Va & Vav & $\mathrm{Va}$ & D & IC & R ou $\mathrm{L}$ & $\mathrm{L}$ \\
\hline TP02 & $\mathrm{Va}$ & Vc & C & V & V & $\mathrm{Va}$ & D & IC & $\mathrm{L}$ & $M$ \\
\hline TP03 & $P$ & Vc & C & V & Vam & $\mathrm{Va}$ & $\mathrm{T}$ & IC & Lp & $M$ \\
\hline TP04 & C & Vc & $\mathrm{Mc}$ & $\mathrm{R}$ & v & Vc & D & IC & $\mathrm{El}$ & $M$ \\
\hline TP05 & C & Vc & $\mathrm{Mc}$ & $\mathrm{R}$ & V & $\mathrm{R}$ & $\mathrm{E}$ & $\mathrm{H}$ & L & $\mathrm{L}$ \\
\hline TP06 & D & Ve & $\mathrm{Mc}$ & V & Vav & $\mathrm{Va}$ & $T$ & IC & R ou $L$ & $M$ \\
\hline XV01 & C & Ve & $\mathrm{Mc}$ & $\mathrm{Va}$ & V & Vc & D & IC & $\mathrm{L}$ & $\mathrm{L}$ \\
\hline XV02 & $P$ & Vc & C & V & Vam & Vc & $\mathrm{T}$ & $\mathrm{H}$ & R ou $L$ & $M$ \\
\hline XV03 & $P$ & Ve & $\mathrm{Me}$ & V & Vam & $\mathrm{Va}$ & $\mathrm{E}$ & IC & R ou $L$ & $M$ \\
\hline XV04 & $\mathrm{Mc}$ & Ve & $\mathrm{Mc}$ & V & v & $\mathrm{Va}$ & $T$ & IC & $\mathrm{L}$ & $M$ \\
\hline XV05 & $\mathrm{Mc}$ & Vc & $\mathrm{Mc}$ & $\mathrm{Va}$ & $\mathrm{R}$ & $\mathrm{Va}$ & $\mathrm{E}$ & $\mathrm{H}$ & L & L \\
\hline XV08 & $P$ & Vc & C & V & Vam & $\mathrm{Va}$ & $T$ & IC & R ou $L$ & $M$ \\
\hline XV09 & $\mathrm{Me}$ & Vc & $\mathrm{Me}$ & Va & V & Vc & $\mathrm{T}$ & $\mathrm{H}$ & $\mathrm{L}$ & L \\
\hline XV10 & C & Vc & $\mathrm{Mc}$ & $\mathrm{Va}$ & Ves & $\mathrm{Va}$ & $\mathrm{T}$ & IC & Lp & $M$ \\
\hline XV11 & C & Ve & $\mathrm{Me}$ & $\mathrm{Va}$ & v & $\mathrm{Va}$ & $T$ & IC & $\mathrm{El}$ & $\mathrm{L}$ \\
\hline RS13 & $P$ & $\mathrm{Ve}$ & $\mathrm{Mc}$ & V & V & Va & $\mathrm{T}$ & IC & $\mathrm{L}$ & $\mathrm{L}$ \\
\hline RS14 & P & Ve & C & V & v & $\mathrm{Va}$ & $\mathrm{T}$ & IC & $\mathrm{L}$ & $M$ \\
\hline
\end{tabular}




\begin{abstract}
*Coloración externa del tallo: $\mathrm{C}=$ Griz, Mc=Marrón claro, $\mathrm{P}=$ Plateado, $\mathrm{D}=$ Dorado, Va=Verde amarillento; Coloración de la corteza: Vc=Verde claro, Ve=Verde oscuro; Coloración de la epidermis: Mc=Marrón claro, C=Crema, Me=Marrón oscuro, La=Naranja;

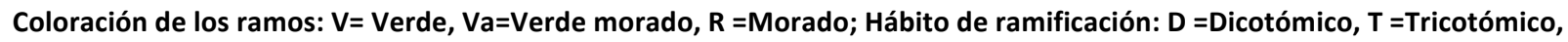
E=Erecto; Posición del peciolo: Ic=Inclinado para arriba, $\mathrm{H}=H$ Horizontal, I=Irregular; Coloración del Peciolo: Ve=Rojo, Va=Verde rojizo, Vam=Verde amarillento, Ves=Rojo verdoso, V=Verde, R=morado; Coloración del rebrote terminal: Va=Verde morado, Vc=Verde claro, R=Morado; Forma del lóbulo: El=Elíptica lanceolada, L=Lanceolada, Lp=Linear Pandurada, R o L=Recta o Linear, Ol=Oblongo lanceolado; Distancia entrenudos: M=Média; L=Larga.
\end{abstract}

\title{
4 CONCLUSIONES
}

Los accesos de yuca recolectados en la Región del Alto Jacuí presentan una elevada variabilidad genética, pasible de ser utilizados en el mejoramiento genético de este cultivo.

Se pudo observar una predominancia del color marrón oscuro en la película de la raíz, del color blanco o crema en la corteza de la raíz, color crema en la pulpa de la raíz y la presencia de pedúnculo sésil en la mayoría de los accesos.

Los materiales estudiados difieren en cuanto a la mayoría de los descriptores morfológicos, con excepción de la pubescencia de la hoja joven y del florecimiento, lo que confirma la posibilidad de uso de estos descriptores para la diferenciación de genotipos en yuca.

\section{AGRADECIMIENTOS}

Al Consejo Nacional de Desarrollo Científico y Tecnológico (CNPq), por el financiamiento por medio de una beca. A la Secretaria de Desarrollo Económico, Ciencia y Tecnología - SDECT-RS y al Banco Mundial por el financiamiento del proyecto. A la Coordinación de Perfeccionamiento de Personal de Nivel Superior (CAPES), a la Fundación de Amparo a la Investigación en el Estado de Rio Grande do Sul (FAPERGS), por las becas de investigación y a la EMATER - RS por su ayuda en la colecta del material genético.

\section{REFERENCIAS}

Alves, A.A.C. (2006) Fisiologia da mandioca. In: EMBRAPA Mandioca e Fruticultura Tropical. Aspectos socioeconômicos e agronômicos da mandioca. Cruz das Almas, BA: EMBRAPA, Brasil, P. 138-169.

Alves-Pereira, A.; Peroni, N.; Abreu, A.G.; Gribel, R.; Clement, C.R. (2011) Genetic structure of traditional varieties of bitter manioc in three soils in central Amazonia. Genetica, v. 139, P. 1259-1271. DOI: 10.1007/s10709-011-9627-4.

Barbosa, C.Z.R.; Alves, J.M.A.; Schwengber, D. R.; Smiderle, O. J. (2007) Características morfológicas e agronômicas de dez clones de mandioca cultivados no estado de Roraima. Agro@mbiente On-line, v. 1, P. 28-31. 
Brito, C.L.L.; Viana, A.E.S.; Barbosa, G. M.; Lopes, S. C.; Santos, V. S.; Silva, V. A. (2013) Caracterização de clones de mandioca (Manihot esculenta Crantz) por meio de descritores morfológicos em Cândido Sales-Bahia. Congresso Brasileiro de Mandioca, 15. Salvador, Brasil.

Chaib, A.M.M.C.; Fialho, J.F.; Vieira, E.A.; Silva, M.S.; Moraes, S.V.; Molovany, J.B.; Paula, G.F.; Souza, F.R.O. (2008) Correlação entre caracteres agronômicos aferidos em acessos do banco regional de germoplasma de mandioca do cerrado. Brasília, DF, Brasil.

Embrapa, Empresa Brasileira de Pesquisa Agropecuária (2006) Centro Nacional de Pesquisa de Solos. Sistema brasileiro de classificação de solos, Brasil.

Enyi, B.A.C. (1972) Effect of shoot number and time of planting on growth, development and yield of cassava (Manihot esculenta Crantz). Journal of Horticultural Science, v. 47, P. 457-456.

Enyi, B.A.C. (1973) Growth rates of three cassava varieties (Manihot esculenta Crantz) under varying population densities. Journal of Agricultural Science, v. 81, P. 15-28.

Faraldo, M.I.F.; Silva, R.M.; Ando, A.; Martins, P.S. (2000) Variabilidade genética de etnovariedades de yuca em regiões geográficas do Brasil. Scientia Agricola, v. 57, P. 499-505.

Fernandes, E.T.; Viana, A.E.S.; Cardoso, A.D.; Cardoso Júnior, N.S.; Lopes, S.C.; Guimarães, D.G.; Anjos, D.N.; Magalhães, G.C.; Fogaça, J.J.N.L. (2009) Caracterização morfológica e produtiva de mandioca variedade periquita cultivada em Vitória da Conquista-BA. In: XIII Congresso Brasileiro de Mandioca, Brasil.

Figueiredo, P.G. (2012) Morfo-anatomia de raízes tuberosas de mandioca (Manihot esculenta Crantz) cultivar IAC 576-70 em diferentes preparos do solo. Dissertação (mestrado). Universidade Estadual Paulista, Faculdade de Ciências Agronômicas. 73p.

Fukuda, W.M.G.; Guevara, C.L. (1998) Descritores morfológicos e agronômicos para a caracterização de yuca (Manihot esculenta Crantz). Cruz das Almas: Embrapa-CNPMF, (Documentos, 78), Brasil.

Gallego, M.R.; Otálvaro, E.V.A.; Peláez, J.C.O.; Monsalve, C.M. (2007) Sustitución de tartrazina por betacaroteno en la elaboración de bebidas no alcohólicas. Revista Lasallista de Investigación, v. 3, P. 7-12.

IBGE, Instituto Brasileiro de Geografia e Estatística. (2017) Levantamento Sistemático da Produção Agrícola. 4p.

Oliveira, N.T.; Alves, J.M.A.; Uchôa, S.C.P.; Rodrigues, G.S.; Melville, C.C.; Albuquerque, J.A.A. (2011) Caracterização e identificação de clones de Mandioca produzidos em Roraima para consumo in natura. Revista Agro@mbiente On line, v. 5, P. 188-193.

Ramos, P.A.S. (2007) Caracterização Morfológica e Produtiva de nove variedades de mandioca cultivadas no Sudoeste da Bahia. Dissertação (Mestrado em Fitotecnia). Universidade Federal de Viçosa, Viçosa, Brasil. 60p.

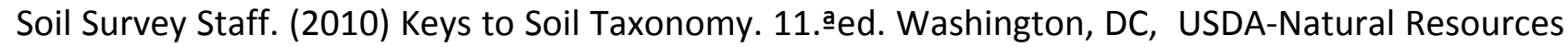
Conservation Service. 338p. 
Takahashi, M.; Guerini, V.L. (1998) Espaçamento para a cultura da mandioca. Brazilian Archives of Biology and Technology, v. 14, P. 489-494.

Tironi, L.F.; UhImann, L.O.; Streck, N.A.; Samboranha, F.K.; Freitas, C.P.O.; Silva, M.R. (2015) Desempenho de cultivares de mandioca em ambiente subtropical. Bragantia, v. 74, P. 58-66.

Vieira, E.A.; Fialho, J.F.; Silva, M.S.; Faleiro, F.G. (2008) Variabilidade genética do banco de germoplasma de mandioca da Embrapa Cerrados acessada por meio de descritores morfológicos. Boletim de pesquisa e desenvolvimento 179, Brasil.

Williams, C. N. (1974). Growth and productivity of tapioca (Manihot utilissima): IV. development and yield of tubers. Experimental Agriculture, v. 10, P. 9-16.

Williams, C.N.; Ghazali, S.M. (1969) Growth and productivity of tapioca (Manihot utilissima): I. leaf characteristics and yield. Experimental Agriculture, v. 5, P. 183-194. 